\title{
Magnetization of a Strongly Interacting Two-Dimensional Electron System in Perpendicular Magnetic Fields
}

\author{
S. Anissimova, A. Venkatesan, A. A. Shashkin, ${ }^{*}$ M. R. Sakr, ${ }^{\dagger}$ and S. V. Kravchenko \\ Physics Department, Northeastern University, Boston, Massachusetts 02115, USA \\ T. M. Klapwijk \\ Kavli Institute of Nanoscience, Delft University of Technology, 2628 CJ Delft, The Netherlands
}

(Received 6 March 2005; published 2 February 2006)

\begin{abstract}
We measure the thermodynamic magnetization of a low-disordered, strongly correlated twodimensional electron system in silicon in perpendicular magnetic fields. A new, parameter-free method is used to directly determine the spectrum characteristics (Landé $g$ factor and the cyclotron mass) when the Fermi level lies outside the spectral gaps and the interlevel interactions between quasiparticles are avoided. Intralevel interactions are found to strongly modify the magnetization, without affecting the determined $g^{*}$ and $m^{*}$.
\end{abstract}

DOI: 10.1103/PhysRevLett.96.046409

PACS numbers: 71.30.+h, 73.40.Qv

Magnetization is one of the least studied properties of two-dimensional (2D) electron systems: signals associated with the magnetization of $2 \mathrm{D}$ electrons are weak, and measuring them is a challenging experiment. Few experimental observations of the de Haas-van Alphen effect in 2D electron systems were made using SQUID magnetometers [1], pick up coils lithographed above the gate [2], or torque magnetometers [3]. A novel method has recently been used by Prus et al. [4] and Shashkin et al. [5] to measure the spin magnetization of 2D electrons in silicon metaloxide-semiconductor field-effect transistors (MOSFETs). This method entails modulating the magnetic field with an auxiliary coil and measuring the imaginary (out-of-phase) component of the ac current induced between the gate and the $2 \mathrm{D}$ electron system, which is proportional to $\partial \mu / \partial B$ (where $\mu$ is the chemical potential). Using the Maxwell relation, $\partial \mu / \partial B=-\partial M / \partial n_{s}$, one can then obtain the magnetization $M$ by integrating the induced current over the electron density, $n_{s}$. Pauli spin susceptibility has been observed to behave critically near the $2 \mathrm{D}$ metal-insulator transition, in agreement with previous transport measurements $[6,7]$.

Here we apply a similar method to study the thermodynamic magnetization of a low-disordered, strongly correlated 2D electron system in silicon MOSFETs in perpendicular and tilted magnetic fields. By measuring $\partial \mu / \partial B$ at noninteger filling factors, we directly determine the spectrum characteristics without any fitting procedures or parameters. As compared to previously used measuring techniques, the remarkable advantage of the novel method is that it probes the spectrum of the 2D electron system with the Fermi level lying outside the spectral gaps so that the effects of interactions between quasiparticles belonging to different energy levels (interlevel interactions) are avoided. Although intralevel interactions are found to strongly affect the magnetization, the extracted Landé $g$ factor and the cyclotron mass are insensitive to them. Therefore, measured spectrum characteristics are likely to be identical with those of a continuous spectrum. The so-obtained $g$ factor has been found to be weakly enhanced and practically independent of the electron density down to the lowest densities reached $\left(\approx 1.5 \times 10^{11} \mathrm{~cm}^{-2}\right)$, while the cyclotron mass becomes strongly enhanced at low $n_{s}$.

Measurements were made in an Oxford dilution refrigerator on clean (100)-silicon samples with peak electron mobilities of $3 \mathrm{~m}^{2} / \mathrm{V} \mathrm{s}$ at $0.1 \mathrm{~K}$ and oxide thickness of $149 \mathrm{~nm}$. Magnetic field $B$ was modulated with a small ac field $B_{\bmod }$ in the range of $0.005-0.03 \mathrm{~T}$ at a low frequency $f=0.05-0.45 \mathrm{~Hz}$ to minimize possible mechanical resonances and avoid overheating the sample. The latter was verified by monitoring the temperature-dependent sample resistance at $B=0$ and in the range of filling factors 1 to 6 with and without modulation. Noticeably higher amplitudes $B_{\text {mod }}$ and/or frequencies $f$ caused overheating of the mixing chamber and were avoided. The in-phase and out-of-phase components of the current between the gate and the 2D electron system were measured with high precision $\left(\sim 10^{-16} \mathrm{~A}\right)$ using a current-voltage converter and a lock-in amplifier. The imaginary (out-of-phase) current component is equal to $\operatorname{Im} i=\left(2 \pi f C B_{\text {mod }} / e\right) d \mu / d B$, where $C$ is the capacitance of the sample. For measurements of the capacitance, a similar circuit was used with a distinction that the gate voltage was modulated and thus the imaginary current component was proportional to the capacitance. The electron density was determined from the capacitance oscillations.

Typical experimental traces of the gate current in a perpendicular magnetic field of $5 \mathrm{~T}$ are displayed in Fig. 1. Sharp dips in the out-of-phase component, seen at integer filling factors $\nu \equiv n_{s} h c / e B_{\perp}$, reflect gaps in the density of states: dips at odd filling factors correspond to the valley splitting, the ones at $\nu=2$ and 6 are due to the 


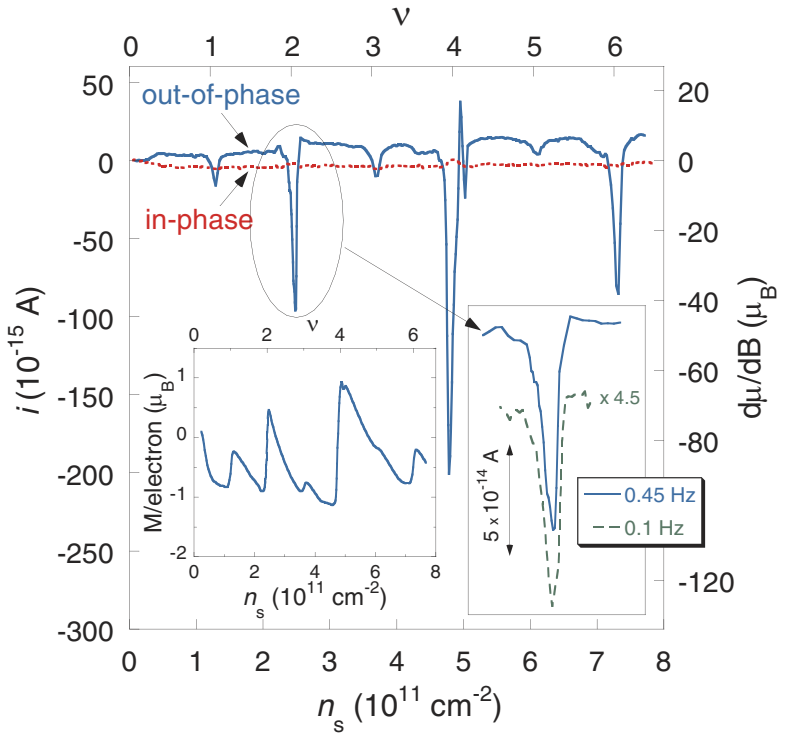

FIG. 1 (color online). Out-of-phase (solid line) and in-phase (dotted line) current components as a function of the electron density in a perpendicular magnetic field of $5 \mathrm{~T}$ and $T=0.8 \mathrm{~K}$. $B_{\text {mod }}=0.022 \mathrm{~T}$ and $f=0.45 \mathrm{~Hz}$. The value $d \mu / d B$ is indicated in units of the Bohr magneton $\mu_{B}$. In the right-hand inset, we demonstrate proportionality of $\operatorname{Im} i$ to frequency: the solid and dashed lines (vertically shifted for clarity) correspond to 0.45 and $0.1 \mathrm{~Hz}$, respectively; the $y$ component of the latter is multiplied by 4.5 . The left-hand inset illustrates magnetization per electron.

spin splitting, and the dip at $\nu=4$ is due to the cyclotron splitting. However, there are no corresponding features in the in-phase current component, which ensures that we reach the low-frequency limit and the measured $\partial \mu / \partial B$ is not distorted by lateral transport effects. This is further confirmed by the fact that the out-of-phase current is proportional to the excitation frequency as displayed in the right-hand inset to Fig. 1. Magnetization per electron can be extracted by integrating the measured out-of-phase signal with respect to $n_{s}$, as shown in the left-hand inset to Fig. 1 for illustration. The magnetization exhibits the expected sawtooth oscillations, with sharp jumps at integer filling factors (note that the height of the jumps yields values that are smaller than the level splitting by the level width).

If the disorder and interactions are disregarded, in quantizing magnetic fields (except at integer filling factors) the derivative $\partial \mu / \partial B=-\partial M / \partial n_{s}$ is equal to

$$
\frac{\partial \mu}{\partial B}=\mu_{B}\left[\left(\frac{1}{2}+N\right) \frac{2 m_{e}}{m_{b}} \pm \frac{1}{2} g_{0}\right]
$$

where $\mu_{B}$ is the Bohr magneton, $N$ is the Landau level number, $m_{e}$ and $m_{b}=0.19 m_{e}$ are the free electron mass and band mass, respectively, and $g_{0}=2$ is the $g$ factor in bulk silicon. Disorder smears out the dependences which otherwise would consist of a series of delta functions. Interactions modify this picture in two ways: (i) by re- normalizing the values of the cyclotron mass and $g$ factor and (ii) by providing a negative contribution of order $-\left(e^{2} / \varepsilon l_{B}\right)\{\nu\}^{1 / 2}$ to the chemical potential [8,9] (here $\varepsilon$ is the dielectric constant, $l_{B}$ is the magnetic length, and $\{\nu\}$ is the deviation of the filling factor from the nearest integer). The latter effect, which is caused by the intralevel interactions between quasiparticles, leads to the so-called negative thermodynamic compressibility near integer filling factors predicted by Efros [8] and experimentally observed in Refs. [10,11].

In Fig. 2, we compare capacitance $C$ with $\partial \mu / \partial B$, measured at the same magnetic field value and plotted versus $n_{s}$ around the filling factor $\nu=2$. The capacitance consists of two contributions: $1 / C=1 / C_{\text {geo }}+1 / A e^{2}\left(d n_{s} / d \mu\right)$, where $C_{\text {geo }}$ is the geometric capacitance [12] depicted by the dashed line in Fig. 2(a), and $A$ is the sample area. (Note that the geometric capacitance slightly increases with $n_{s}$ since the thickness of the 2D electron layer-and, therefore, the average distance between the 2D layer and the gate-decreases with the gate voltage.) The second term is responsible for the dip centered at $n_{s}=3.87 \times 10^{11} \mathrm{~cm}^{-2}$, corresponding to $\nu=2$, and sharp maxima on both sides of it. Note that at these maxima, the capacitance exceeds $C_{\text {geo }}$, which corresponds to the negative thermodynamic compressibility discussed above. Farther from integer filling factors, the intralevel interaction corrections become weak, being proportional to $\{\nu\}^{-1 / 2}$, and the measured capacitance approaches $C_{\text {geo }}$ (as long as the broadening of Landau levels is negligible, i.e., $d n_{s} / d \mu \gg d n_{s} /\left.d \mu\right|_{B=0}$ ).

Similar maxima on both sides of $\nu=2$ are seen in the magnetization data shown in Fig. 2(b). At the maxima, the derivative $\partial \mu / \partial B$ exceeds maximum values possible in a noninteracting $2 \mathrm{D}$ electron gas, which are determined by Eq. (1) and are depicted in the figure by dashed lines. The possibility that $\partial \mu / \partial B$ might exceed its maximum noninteracting values due to intralevel Coulomb interactions between quasiparticles was predicted by MacDonald et al. [9]; in fact, this is how negative compressibility [8] manifests itself in magnetization measurements. The sharp
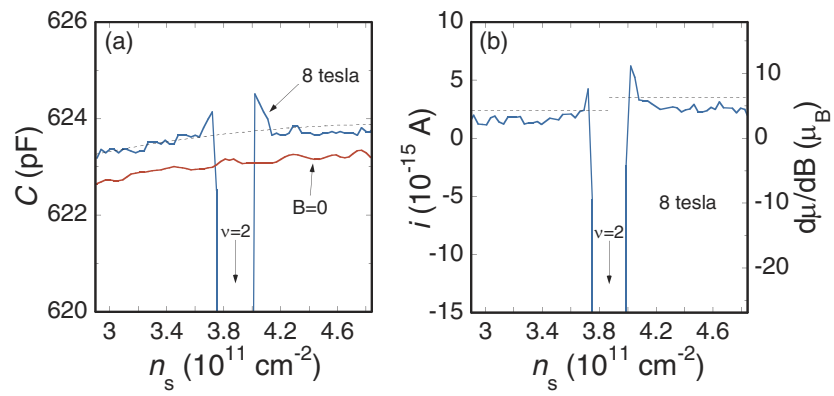

FIG. 2 (color online). (a) Capacitance in $B=8 \mathrm{~T}$ and in $B=$ 0 as indicated. The (noise-averaged) geometric capacitance is depicted by a dashed line. (b) $\operatorname{Im} i \propto d \mu / d B$ in a perpendicular magnetic field of $8 \mathrm{~T}$. The maximum values possible in a noninteracting system (see text) are depicted by dashed lines. 
spike just above $\nu=4$ and maxima on both sides of $\nu=2$ in the dependence shown in Fig. 1 are of the same nature.

It is straightforward to obtain the effective $g$ factor from the data for $\partial \mu / \partial B$. In accordance with Eq. (1), it is equal (in units of the Bohr magneton) to the difference between $\partial \mu / \partial B$ for spin-down $(\downarrow)$ and spin-up $(\uparrow)$ electrons belonging to the same Landau level: $\mu_{B} g^{*}=(\partial \mu / \partial B)_{\downarrow}-$ $(\partial \mu / \partial B)_{\uparrow}$. It is important that this method of determining the $g$ factor does not require the use of any fitting procedures or parameters. Figs. 3(a) and 3(b) show measured $\partial \mu / \partial B$ as a function of the deviation of the filling factor from 2 at two values of magnetic field. Near $\nu=2$, there are sharp intralevel interaction-induced structures discussed above; these regions have been excluded from the analysis. However, farther from $\nu=2$, the dependences for $\nu<2$ and $\nu>2$ become parallel to each other. This ensures that the so-determined $g^{*}$ is not affected by the valley splitting $[13,14]$ and intralevel interaction effects $[8,9]$ discussed above. The latter contribute equally to both spin-up and spin-down dependences and cancel each other out. Disorder also contributes equally to $\partial \mu / \partial B$ on both sides of $\nu=2$ : we have found that at magnetic fields down to approximately $3 \mathrm{~T}$, there are wide regions of filling factors where capacitance (i.e., the density of states) is symmetric around $\nu=2$ [see, e.g., Fig. 2(a)]; furthermore, closeness of the capacitance to $C_{\mathrm{geo}}$ attests that the disorder-induced corrections are small. At lower magnetic fields, however, the electron-hole symmetry around $\nu=2$ breaks down, which sets the lower boundary for the range of magnetic fields (and, consequently, electron densities). Note that temperature smears out the dependences in a way similar to disorder: at higher temperatures, the capacitance at half-integer filling factors decreases, which leads to a worsening of the method accuracy.

In Fig. 4 we plot the measured $g$ factor along with the one previously obtained from transport measurements (solid line). One can see that there is no systematic dependence of the $g$ factor on $n_{s}$ : it remains approximately constant and close to its value in bulk silicon even at the lowest electron densities, which is in good agreement with the transport [15] and magnetocapacitance [13] results.
The same method can be used for determination of the cyclotron mass in tilted magnetic fields strong enough to completely polarize the electron spins [16]. If (and only if) the spin splitting exceeds the cyclotron splitting, the gap at $\nu=2$ lies between Landau levels $0 \uparrow$ and $1 \uparrow$, and the difference $(\partial \mu / \partial B)_{N=1}-(\partial \mu / \partial B)_{N=0}$ is equal to $2 \mu_{B}\left(m_{e} / m^{*}\right) \cos \phi$, where $\phi$ is the tilt angle. Once the electron spins are fully polarized at filling factors above $\nu=2$, the tilt angle is automatically large enough for the level crossing to have occurred. The region of explorable electron densities is restricted from above by the condition that the electrons must be fully spin-polarized, while with our current setup, the maximum magnetic field at which we can apply the modulation is only $8 \mathrm{~T}$ capable of polarizing the electron spins up to $n_{s}^{*} \approx 2 \times 10^{11} \mathrm{~cm}^{-2}[5,17]$. Figures 3(c) and 3(d) show $\partial \mu / \partial B$ as a function of $|\nu-2|$ under the condition $n_{s}<n_{s}^{*}$ at two tilt angles [18]. The extracted cyclotron mass at electron densities 1.55 and $1.35 \times 10^{11} \mathrm{~cm}^{-2}$ is significantly enhanced. At densities below $1.35 \times 10^{11} \mathrm{~cm}^{-2}$, the symmetry of capacitance on both sides of the $\nu=2$ gap breaks down, making the determination of $m^{*}$ impossible. As a result, we were only able to obtain two data points. Nevertheless, good agreement with the effective mass previously obtained by transport measurements (Fig. 4) demonstrates the applicability of the new method and adds credibility to both transport and magnetization results.

We stress once again that the advantage of the new method we use here is that it allows determination of the spectrum of the 2D electron system under the condition that the Fermi level lies outside the spectral gaps, and the interlevel interactions are avoided. Being symmetric about $\nu=2$, the intralevel interactions are canceled out in the data analysis and do not influence the extracted $g$ factor and cyclotron mass. Therefore, the obtained values $g^{*}$ and $m^{*}$ are likely to be identical with those for a continuous spectrum, and the comparison with previously found values of the $g$ factor and the effective mass is valid.

To summarize, thermodynamic magnetization measurements in perpendicular and tilted magnetic fields allow determination of the spectrum characteristics of $2 \mathrm{D}$ elec-
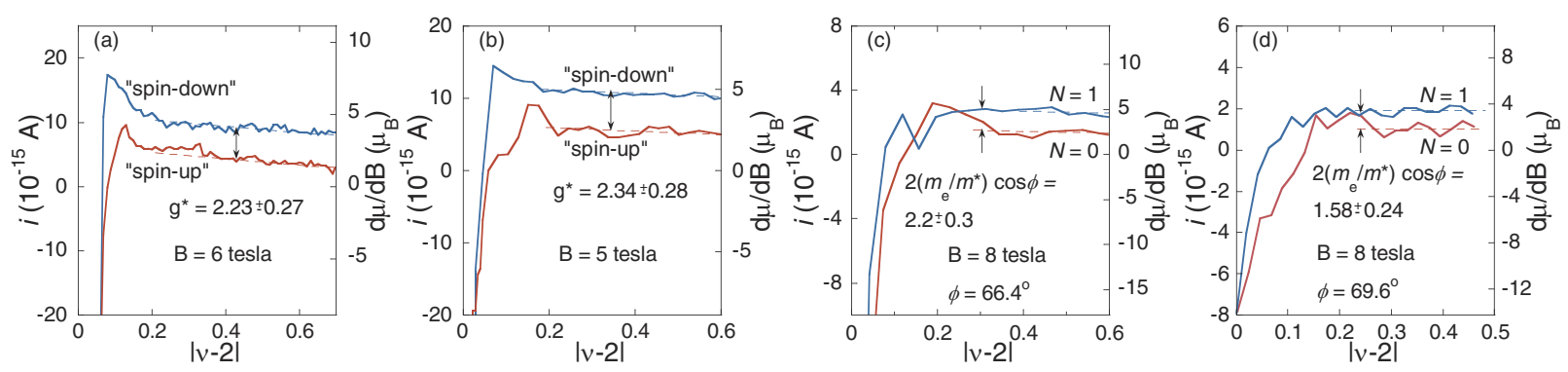

FIG. 3 (color online). Illustration of how the effective $g$ factor (a),(b) and the cyclotron mass (c),(d) have been measured. The imaginary current component is plotted as a function of the deviation of the filling factor from $\nu=2$. In perpendicular magnetic fields, the difference between $\partial \mu / \partial B$ for spin-down $(\downarrow)$ and spin-up $(\uparrow)$ electrons yields $g^{*}$ in units of the Bohr magneton. In tilted magnetic fields, the difference between $\partial \mu / \partial B$ for electrons with $N=1$ and $N=0$ is equal to $2 \mu_{B}\left(m_{e} / m^{*}\right) \cos \phi$. The dashed lines show noiseaveraged values. $B_{\text {mod }}=0.022 \mathrm{~T}(\mathrm{a}),(\mathrm{b})$ and $0.0055 \mathrm{~T}(\mathrm{c}),(\mathrm{d})$. 


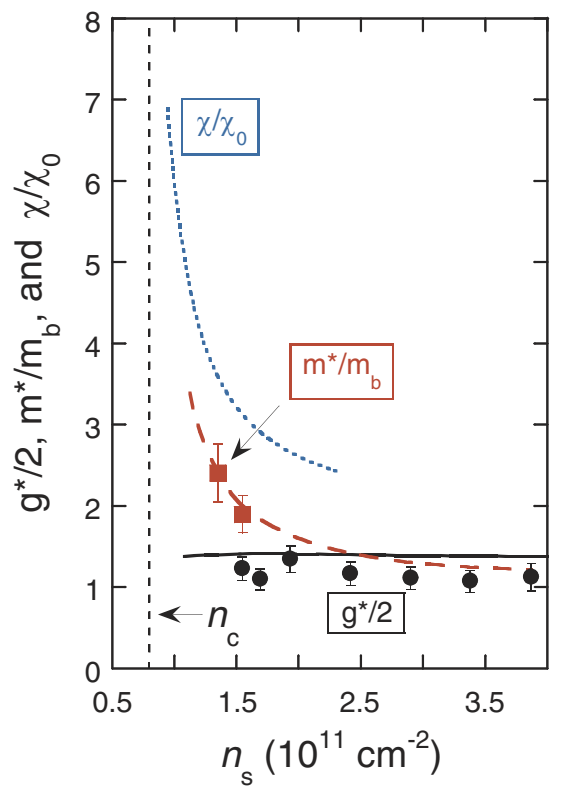

FIG. 4 (color online). The effective $g$ factor (circles) and the cyclotron mass (squares) as a function of the electron density. The solid and long-dashed lines represent, respectively, the $g$ factor and effective mass, previously obtained from transport measurements [15], and the dotted line is the Pauli spin susceptibility obtained by magnetization measurements in parallel magnetic fields [5]. The critical density $n_{c}$ for the metal-insulator transition is indicated.

tron systems and show that enhancement of the $g$ factor is weak and practically independent of the electron density, while the cyclotron mass becomes strongly enhanced as the density is decreased. The obtained data agree well with the $g$ factor and effective mass obtained by transport measurements, as well as with the Pauli spin susceptibility obtained by magnetization measurements in parallel magnetic fields, even though the lowest electron densities reached in the experiment are somewhat higher. Thus, we arrive at the conclusion that, unlike in the Stoner scenario, it is indeed the effective mass that is responsible for the dramatically enhanced spin susceptibility at low electron densities.

We gratefully acknowledge discussions with V.T. Dolgopolov, B.I. Halperin, and M.P. Sarachik. This work was supported by the National Science Foundation Grant No. DMR-0403026, the ACS Petroleum Research Fund Grant No. 41867-AC10, the RFBR, RAS, and the Programme "The State Support of Leading Scientific Schools."

Note added in proof. - After this work had been completed, we learned that Punnoose and Finkelstein [19] made a renormalization group analysis for multivalley 2D systems. Their conclusion that the effective mass dramatically increases at the metal-insulator transition while the $g$ factor remains nearly intact is consistent with our experimental results.
*Permanent address: Institute of Solid State Physics, Chernogolovka, Moscow District 142432, Russia.

${ }^{\dagger}$ Present address: Department of Physics and Astronomy, UCLA, Los Angeles, CA 90095, USA.

[1] H. Stormer, T. Haavasoja, V. Narayanamurti, A.C. Gossard, and W. Wiegmann, J. Vac. Sci. Technol. B 1, 423 (1983); I. Meinel, D. Grundler, S. Bargstadt-Franke, C. Heyn, and D. Heitmann, Appl. Phys. Lett. 70, 3305 (1997).

[2] F. F. Fang and P. J. Stiles, Phys. Rev. B 28, 6992 (1983).

[3] J.P. Eisenstein, Appl. Phys. Lett. 46, 695 (1985); J. P. Eisenstein, H. L. Stormer, V. Narayanamurti, A. Y. Cho, A. C. Gossard, and C. W. Tu, Phys. Rev. Lett. 55, 875 (1985); S. A. J. Wiegers, M. Specht, L. P. Levy, M. Y. Simmons, D. A. Ritchie, A. Cavanna, B. Etienne, G. Martinez, and P. Wyder, Phys. Rev. Lett. 79, 3238 (1997); M. Zhu, A. Usher, A. J. Matthews, A. Potts, M. Elliott, W. G. Herrenden-Harker, D. A. Ritchie, and M. Y. Simmons, Phys. Rev. B 67, 155329 (2003).

[4] O. Prus, Y. Yaish, M. Reznikov, U. Sivan, and V. Pudalov, Phys. Rev. B 67, 205407 (2003).

[5] A. A. Shashkin, S. Anissimova, M. R. Sakr, S. V. Kravchenko, V.T. Dolgopolov, and T.M. Klapwijk, Phys. Rev. Lett. 96, 036403 (2006).

[6] S. V. Kravchenko and M. P. Sarachik, Rep. Prog. Phys. 67, 1 (2004).

[7] A. A. Shashkin, Phys. Usp. 48, 129 (2005).

[8] A. L. Efros, Solid State Commun. 65, 1281 (1988).

[9] A.H. MacDonald, H.C.A. Oji, and K.L. Liu, Phys. Rev. B 34, 2681 (1986).

[10] S. V. Kravchenko, V. M. Pudalov, and S. G. Semenchinsky, Phys. Lett. A 141, 71 (1989).

[11] J. P. Eisenstein, L. N. Pfeiffer, and K. W. West, Phys. Rev. Lett. 68, 674 (1992).

[12] The geometric capacitance has been determined according to $1 / C_{\text {geo }}=1 /\left.C\right|_{B=0}-1 /\left.A e^{2}\left(d n_{s} / d \mu\right)\right|_{B=0}$ (for more on this procedure, see Ref. [13]).

[13] V. S. Khrapai, A. A. Shashkin, and V. T. Dolgopolov, Phys. Rev. Lett. 91, 126404 (2003); Phys. Rev. B 67, 113305 (2003).

[14] The bare valley splitting is independent of the magnetic field and does not contribute to $\partial \mu / \partial B$. However, it may be enhanced by interlevel interactions [13]. The fact that $\partial \mu / \partial B$ for $\nu<2$ and $\nu>2$ are parallel to each other ensures that a possible influence of the enhanced valley splitting is negligible in our experiment.

[15] A. A. Shashkin, S. V. Kravchenko, V. T. Dolgopolov, and T. M. Klapwijk, Phys. Rev. B 66, 073303 (2002).

[16] The effects of finite layer thickness, which lead to an increase of the effective mass with parallel magnetic field, are negligible in silicon MOSFETs [6,7].

[17] S. A. Vitkalov, H. Zheng, K. M. Mertes, M. P. Sarachik, and T. M. Klapwijk, Phys. Rev. Lett. 85, 2164 (2000); A. A. Shashkin, M. Rahimi, S. Anissimova, S. V. Kravchenko, V.T. Dolgopolov, and T.M. Klapwijk, Phys. Rev. Lett. 91, 046403 (2003).

[18] Using $n_{s}^{*}(8 \mathrm{~T}) \approx 2 \times 10^{11} \mathrm{~cm}^{-2}$, one can estimate that the coincidence of spin and cyclotron splittings for $\nu=$ 2 occurs at $\phi^{*}=\cos ^{-1}\left(n_{s}^{*} h c / 2 e B\right) \approx 59^{\circ}$.

[19] A. Punnoose and A. M. Finkelstein, Science 310, 289 (2005). 